\title{
Smelling Sounds: Olfactory-Auditory Sensory Convergence in the Olfactory Tubercle
}

\author{
Daniel W. Wesson ${ }^{1,2}$ and Donald A. Wilson ${ }^{1,2}$ \\ ${ }^{1}$ Emotional Brain Institute, Nathan S. Kline Institute for Psychiatric Research, Orangeburg, New York 10962, and ${ }^{2}$ Department of Child and Adolescent \\ Psychiatry, New York University School of Medicine, New York, New York 10016
}

\begin{abstract}
Historical and psychophysical literature has demonstrated a perceptual interplay between olfactory and auditory stimuli-the neural mechanisms of which are not understood. Here, we report novel findings revealing that the early olfactory code is subjected to auditory cross-modal influences. In vivo extracellular recordings from the olfactory tubercle, a trilaminar structure within the basal forebrain, of anesthetized mice revealed that olfactory tubercle single units selectively respond to odors-with $65 \%$ of units showing significant odor-evoked activity. Remarkably, $19 \%$ of olfactory tubercle single units also showed robust responses to an auditory tone. Furthermore, $29 \%$ of single units tested displayed supraadditive or suppressive responses to the simultaneous presentation of odor and tone, suggesting cross-modal modulation. In contrast, olfactory bulb units did not show significant responses to tone presentation nor modulation of odor-evoked activity by tone-suggesting a lack of olfactory-auditory convergence upstream from the olfactory tubercle. Thus, the tubercle presents itself as a source for direct multimodal convergence within an early stage of odor processing and may serve as a seat for psychophysical interactions between smells and sounds.
\end{abstract}

\section{Introduction}

The integration of environmental information across multiple sensory channels is critical to guiding decisions and behaviors. For instance, whereas the sound of a car horn while crossing a street may evoke arousal, the sound of the same horn matched with the sight of a rapidly approaching taxicab may result in your quickly getting out of the street. A basic tenet of multisensory integration is the ability of one sensory modality to enhance or to suppress information from another sensory modality (Calvert et al., 2004). This is distinct from modulatory interactions such as those occurring during dishabituation (Rankin et al., 2009; Smith et al., 2009) or fear-potentiated responses (Davis, 1989; Halene et al., 2009) in which an intense stimulus in one modality may enhance responsiveness to stimuli in another-as a result of systemwide changes in neural excitability. Rather, multisensory integration involves convergence of sensory pathways, often with single neurons displaying stimulus-evoked activity to both, and/or modification of the response to one sensory input while in the context of another.

Although most information on the neural basis of multisensory integration stems from studies in somatosensation, audition, and vision, the olfactory system is known to interact with at least two sensory systems. For instance, the percept of "flavor" is a multimodal construct resultant from cellular interactions between olfactory and gustatory processing centers (Verhagen and Engelen, 2006). Furthermore, it has been shown that visual cues

Received Dec. 4, 2009; accepted Jan. 4, 2010.

This work was supported by National Institutes of Health Grant DC003906 (D.A.W.).

Correspondence should be addressed to Daniel W. Wesson, Emotional Brain Institute, Nathan S. Kline Institute for

Psychiatric Research, Orangeburg, NY 10962. E-mail: dwesson@nki.rfmh.org.

DOI:10.1523/JNEUROSCI.6003-09.2010

Copyright $\odot 2010$ the authors $\quad 0270-6474 / 10 / 303013-09 \$ 15.00 / 0$ may facilitate odor detection (Gottfried and Dolan, 2003). Integration of olfactory information with other sensory channels could have significance for a variety of critical scenarios (e.g., food selection and threat aversion).

The present study stems from a serendipitous observation of tone-evoked single-unit responses in the olfactory tubercle. However, $>150$ years ago, the perfumer G. W. Piesse suggested that olfactory perception may be intimately linked with auditory processing (Piesse, 1857). In particular, Piesse postulated a strategy to catalog odors based on analogous auditory pitches. A recent psychophysical test of this concept by Belkin et al. (1997) showed that human subjects are readily capable of consistently matching odor qualities to auditory pitch. Furthermore, although rare, olfactory-auditory synesthesia has been reported in humans (Simpson and McKellar, 1955). Thus, a perceptual relationship between olfactory and auditory perception is apparent, although no neural evidence of direct olfactory-auditory convergence has been reported to our knowledge. Such convergence of olfactory and auditory channels likely requires a site wherein projections are received directly or indirectly from both principal olfactory and auditory areas.

One potential region of interest in olfactory-auditory interactions is the understudied and enigmatic olfactory tubercle. The olfactory tubercle is a trilaminar cortical-like structure that occupies a large portion of the basal forebrain. Previous anatomical work has shown that the tubercle receives monosynaptic olfactory input from the olfactory bulb (OB) and the olfactory piriform cortex (PCX) (Haberly and Price, 1977; Schwob and Price, 1984; Johnson et al., 2000). Very recent work showed that electrical stimulation of the lateral olfactory tract (LOT) evokes neural responses in the olfactory tubercle (Carriero et al., 2009). Additionally, auditory sensory information may arrive at the ol- 
factory tubercle via general associative networks involving the hippocampus (Deadwyler et al., 1987) or ventral pallidum (Budinger et al., 2008) — both regions being interconnected with the olfactory tubercle (Ikemoto, 2007). Alternatively, auditory information from the primary auditory cortex may converge with olfactory information in the olfactory tubercle directly (Budinger et al., 2006). Although the olfactory tubercle is positioned anatomically in a manner that supports a potential role in olfactory-auditory sensory integration, to our knowledge, no studies have tested the response to both odors and auditory stimuli there. Therefore, here, we tested the role of the olfactory tubercle in olfactory-auditory sensory convergence.

\section{Materials and Methods}

Experimental subjects. Twenty-three adult male BL6 mice (2-6 months of age) bred and maintained within the Nathan S. Kline Institute for Psychiatric Research animal facility were used. Food and water were available ad libitum. Mice were housed on a $12 \mathrm{~h}$ light/dark cycle with all experiments performed during the light phase. All experiments were conducted in accordance with the guidelines of the National Institutes of Health and were approved by the Institutional Animal Care Committee of the Nathan S. Kline Institute.

Electrophysiology. For in vivo electrophysiology, mice were anesthetized with urethane anesthesia $(2.0 \mathrm{mg} / \mathrm{kg}$, i.p. $)$ and supplied with atropine hydrochloride $(25 \mathrm{mg} / \mathrm{kg}$, i.m.) to minimize tracheal congestion. Mice were then mounted in a stereotaxic frame outfitted with a waterfilled heating pad $\left(38^{\circ} \mathrm{C}\right)$, and the skin overlying the skull was administered local anesthetic ( $1 \%$ xylocaine, s.c.) and later removed, exposing the dorsal skull. To prevent closure of the ear canal, stereotaxic ear bars were secured within the posterior aspects of the orbital sockets for all preparations. Small ( $\sim 1.5$-mm-diameter) ipsilateral holes were drilled over the olfactory tubercle and/or the $\mathrm{OB}$ and LOT. A tungsten stimulating electrode was lowered into the mesial region of the $\mathrm{OB}$ granule cell layer to aid in tubercle localization under physiological control by OB stimulation. Likely because of the gyrating anatomy of the tubercle, clear OB stimulation-evoked positive and negative evoked potentials that are apparent in the piriform cortex, were sometimes difficult to determine. In a subset of preparations ( $n=5$ mice), we performed recordings from mitral/tufted cells (MTs) of the OB. For these recordings, the stimulating electrode was lowered to the LOT to aid in MT localization under physiological control by LOT stimulation. Electrode locations were verified with postmortem histology using cresyl violet staining of slide-mounted 40 $\mu \mathrm{m}$ coronal sections. As shown in Figure 1, electrode tips spanned olfactory tubercle layers I through III. Preparations in which the electrode tips were found outside of the tubercle were entirely excluded from this study. Recording electrode potentials along with stimulus presentation events were acquired using Spike2 software (Cambridge Electronic Design).

Stimulus presentation. Odors were presented to anesthetized mice using an air dilution olfactometer (Rennaker et al., 2007) at $1 \mathrm{~L} / \mathrm{min}$ flow using medical-grade nitrogen. Stimuli included five monomolecular odorants and mixtures of monomolecular odorants as previously described (Barnes et al., 2008). The five monomolecular stimuli included 1,7-octadiene, 4-methyl-3-penten-2one, ethyl propionate, heptanal, and isoamyl acetate (Sigma-Aldrich). All odorants were "pure" in their liquid state except for 1,7-octadiene and 4-methyl-3-penten-2-one, which were diluted 1:1 in mineral oil. Odor mixtures were based off of previous work in our laboratory using 10

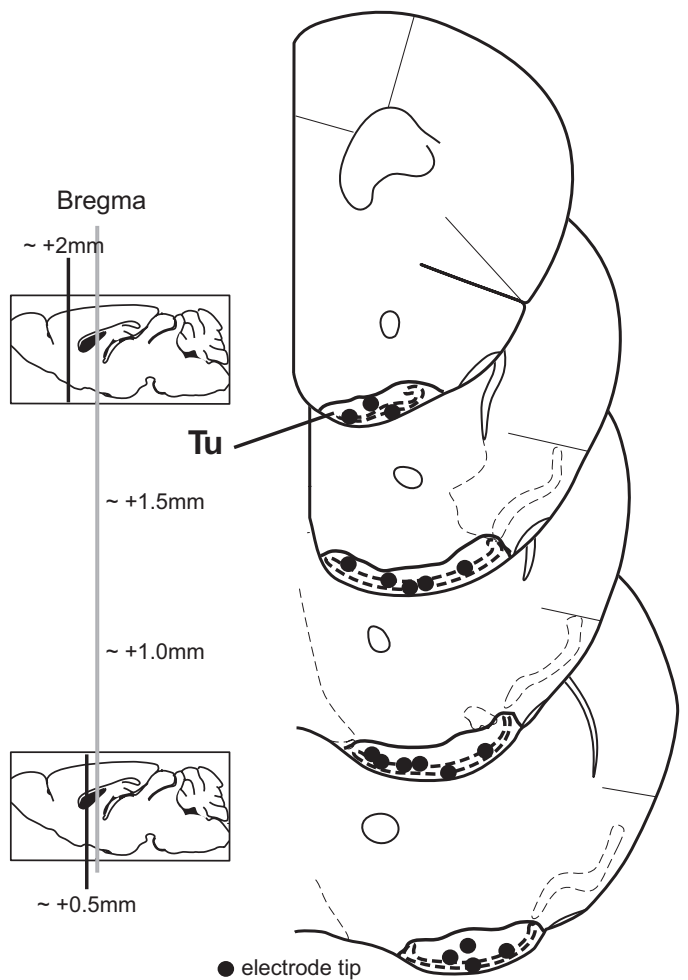

Figure 1. Electrode placement in the tubercle. Coronal stereotaxic panels showing the approximatelocation of electrode tips (black dots) from 18 mice in theolfactory tubercle (Tu). Coronal sections span from 2.0 to $0.5 \mathrm{~mm}$ anterior of bregma, in $0.5 \mathrm{~mm}$ intervals. The region outlined in bold black is Tu. The panels are adapted from the work by Paxinos and Franklin (2000).

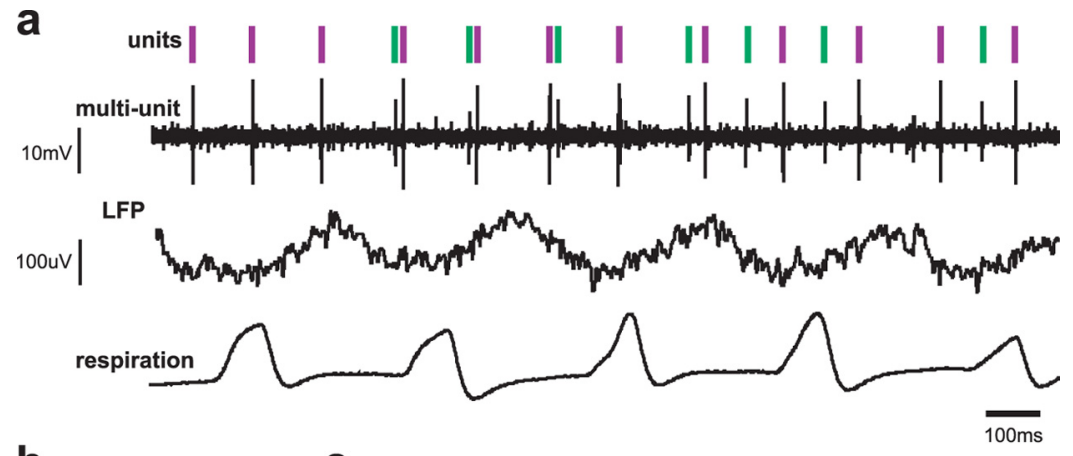

b
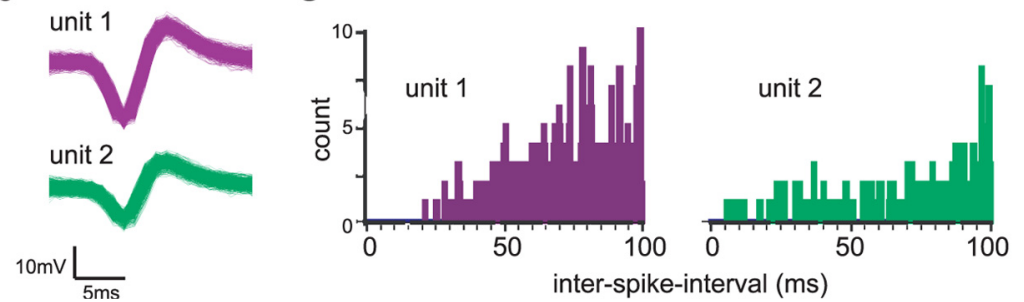

Figure 2. In vivo spontaneous multiunit tubercle activity in anesthetized mice. $\boldsymbol{a}$, Representative traces showing multiunit activity, LFP (100 Hz low-pass filter), and respiration over the course of $2 \mathrm{~s}$. Inhalation in respiratory trace is an upward deflection. The hash marks represent unit responses (spikes). $\boldsymbol{b}$, Waveform plots for two units (unit 1, top; unit 2, bottom) indicated in $\boldsymbol{a}$ by purple and green hash marks, respectively. $\boldsymbol{c}$, Interspike interval distributions for units displayed in $\boldsymbol{a}$ and $\boldsymbol{b}$. Note for each unit there were no spikes with intervals $\leq 2 \mathrm{~ms}$. 
before mixing. Odors were presented $2 \mathrm{~s}$ each, at a minimal $30 \mathrm{~s}$ interstimulus interval (ISI), and were triggered off of the animal's respiration using a piezo foil placed under the animal's chest and a window discriminator to detect peaks of respiration (World Precision Instruments).

Auditory ("tone") stimuli were presented using a $76 \mathrm{~dB}, 2.8 \mathrm{kHz}$ piezo speaker (model 273-059; Radio Shack), located $\sim 30 \mathrm{~cm}$ directly anterior to the mouse (on the exterior of a faraday cage). The speaker was powered by a $5 \mathrm{~V}$ digital output from the CED data acquisition and control board. Tone onset was triggered off of the animal's respiratory signal in the same way as done for odor stimuli. Tones were presented $2 \mathrm{~s}$ each, at a minimal 30 s ISI. All stimuli were presented for at least five counterbalanced trials for each unit (range, 5-16 trials/unit).

Data analysis. Electrophysiological data were analyzed as previously described (Wilson, 1998; Rennaker et al., 2007). Single-unit spike sorting, cluster cutting, and waveform analysis were all performed in Spike2 software (Cambridge Electronic Design). Figure 2, $a$ and $b$, shows example spikes, single-unit identification, and waveforms of two single units, isolated from an individual olfactory tubercle recording. Also, the recordings were verified as single units by a conservative interspike interval threshold. No more than $1 \%$ of spikes from a single unit could occur with an interspike interval of $<2 \mathrm{~ms}$. Example waveforms and interspike interval distributions for two units are shown in Figure 2, $b$ and $c$. Putative units that did not pass these criteria were omitted from additional analysis. Both MT and olfactory tubercle units underwent the same sorting and analysis methods.

To determine responsiveness of units to stimuli, the spike magnitude (total number of spikes) during both the $2 \mathrm{~s}$ prestimulus and the $2 \mathrm{~s}$ during stimulus were pooled across trials for each individual stimulus. Paired two-tailed $t$ tests were then performed to test for an evoked response. In this manner, a unit is said to be significantly modulated by a stimulus when, across all trials, there was a significant $(p>0.05)$ effect of stimulus presentation on the spike rate. All statistical analyses were performed in StatVIEW (SAS Institute) or in MATLAB (The MathWorks). All values are reported as mean \pm SEM unless otherwise stated.

\section{Results}

\section{Odor-evoked responses in the olfactory tubercle}

To begin elucidating the potential contributions of the olfactory tubercle to the multimodal convergence of odors and sounds, we recorded from a total of 62 confirmed olfactory tubercle single units in urethane-anesthetized mice (1-3 units/mouse) in response to either odors, tones, or both. The majority of olfactory tubercle single units recorded were spontaneously active, with a $14.1 \pm 15 \mathrm{~Hz}$ (mean $\pm \mathrm{SD}$ ) spontaneous firing rate across all units (Fig. 3a). However, the mean was biased by several veryhigh-frequency single units as most cells recorded from had a relatively low spontaneous firing rate (mode, $<5 \mathrm{~Hz}$ ) (Fig. $3 a$ ). Neural activity at both the population [local field potential (LFP)] and single-unit level were generally phasic with respiration-with the phasic component of each response beginning momentarily after inspiration (Fig. $3 b$ ). Presentation of an odorant altered the LFP and spontaneous firing rate. In the example shown in Figure $3 b$, the odorant 1,7-octadiene evoked both a transformation of the LFP and two action potentials on the first inhalation. In this particular example, subsequent inhalations of the odorant evoked LFP oscillations and unit action potentials until two inhalations after odorant offset (likely reflecting lingering of the odorant around the nostrils). The odor-evoked LFP responses were qualitatively similar to those seen previously in the olfactory system (Freeman, 1978; Cenier et al., 2008; Kay et al., 2009) and consisted of beta (10-35 Hz) and gamma band $(40-70 \mathrm{~Hz})$ high-frequency oscillations riding on the downward crest of an enhanced theta $(1-10 \mathrm{~Hz})$ rhythm (Fig. 3b, $0-100 \mathrm{~Hz}$ trace). LFP power spectrum analyses (paired $t$ tests for $1 \mathrm{~Hz}$ bins) confirmed that odorant presentation evoked significant changes in theta $\left(t_{(9)}=-3.45 ; p<0.01\right)$, beta $\left(t_{(24)}=-8.04 ; p<0.0001\right)$,
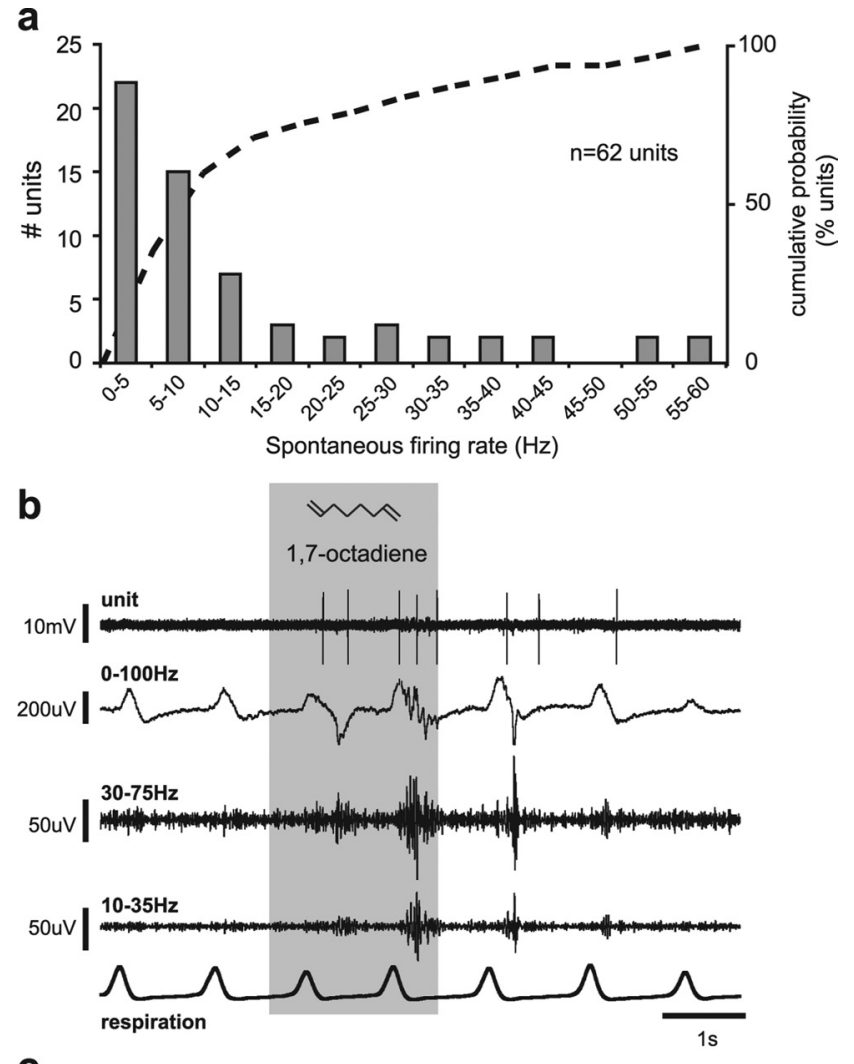

C

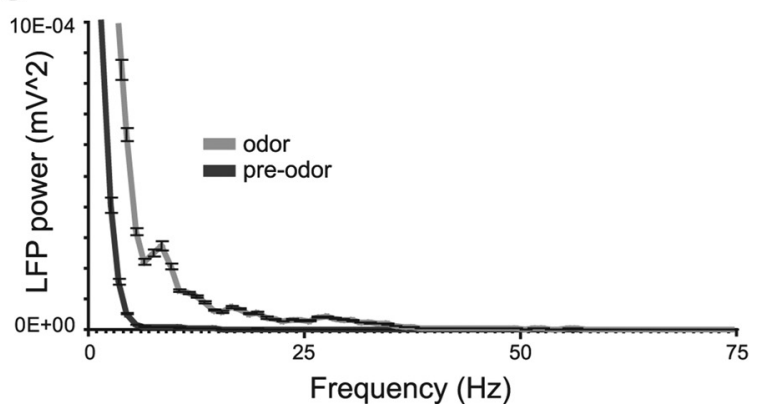

Figure 3. Odorant presentation evokes neural responses in the tubercle. $\boldsymbol{a}$, Histogram (bars) and cumulative probability plot (dashed line) showing spontaneous firing rate distributions (in hertz) of 62 tubercle units. Spontaneous firing rate, Average instantaneous firing rate during the 5 s before all stimulus presentations for each unit. $\boldsymbol{b}$, "Unit" trace showing multiunit activity, LFPs filtered from $0-100,30-75$ (gamma), and $10-35 \mathrm{~Hz}$ (beta) (second-order bandpass) and respiration. Inhalation in respiratory trace is an upward deflection. 0dor presentation (1,7-octadiene; gray shaded region) evoked high-frequency LFP oscillations and action potentials. Note that action potentials and LFP oscillations were generally phasic and closely follow respiratory peaks. c, Power spectrograms of LFP activity before (preodor; 2 s before odor) and during (odor; 2 s during odor) odor presentation. Data are an average from four mice, four odors each, and two trials per odor. Odor presentation evoked changes in theta $(0-10 \mathrm{~Hz} ; p<0.0001$, two-tailed $t$ test), beta ( $10-35 \mathrm{~Hz}$; $p<0.0001$, two-tailed $t$ test), and gamma ( $30-75 \mathrm{~Hz} ; p<0.0001$, two-tailed $t$ test) oscillations compared with preodor. Error bars indicate SEM.

and gamma $\left(t_{(28)}=-8.13 ; p<0.0001\right)$ oscillatory activity (Fig. $3 c$ ). These results demonstrate, as predicted by both anatomical (Haberly and Price, 1978; Scott et al., 1980; Schwob and Price, 1984) and physiological studies (Murakami et al., 2005; Chiang and Strowbridge, 2007; Carriero et al., 2009), that the olfactory tubercle responds to odors at both the population and single-unit level.

Previous work has demonstrated that mitral-tufted cells in the OB (Wilson and Leon, 1988; Kay and Laurent, 1999; Cang and Isaacson, 2003; Nagayama et al., 2004; Rinberg et al., 2006; Davison 
a
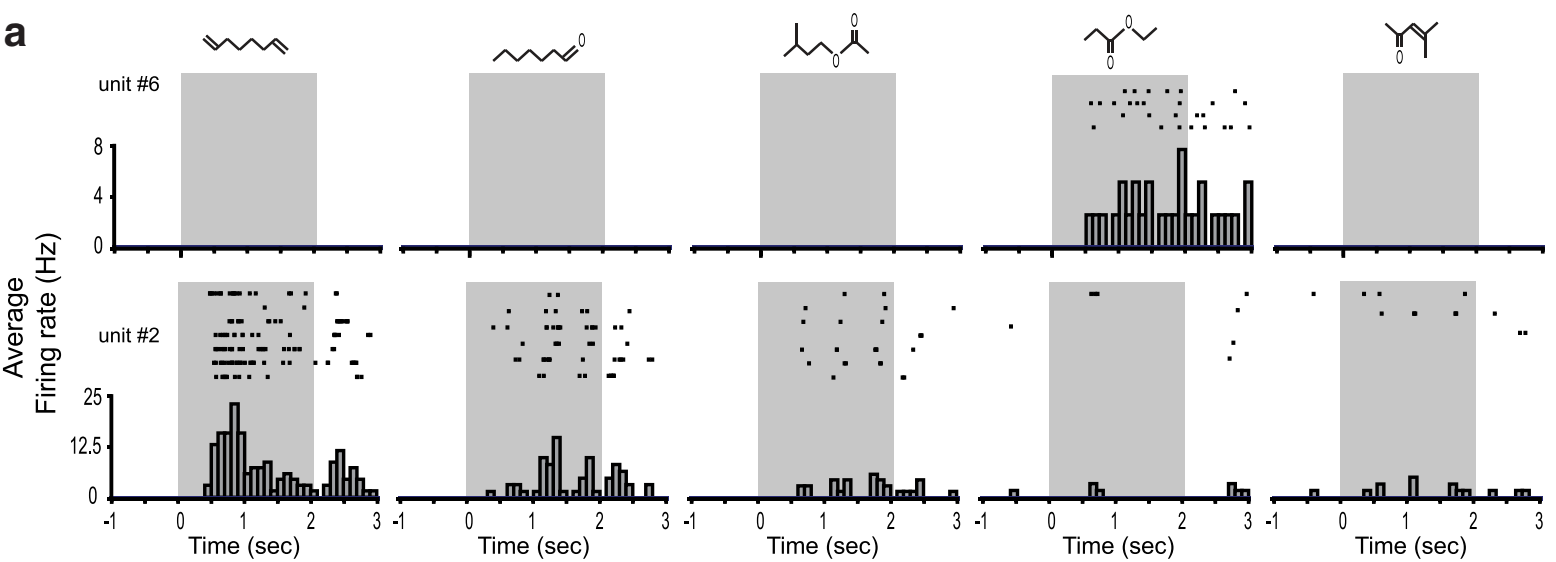

b

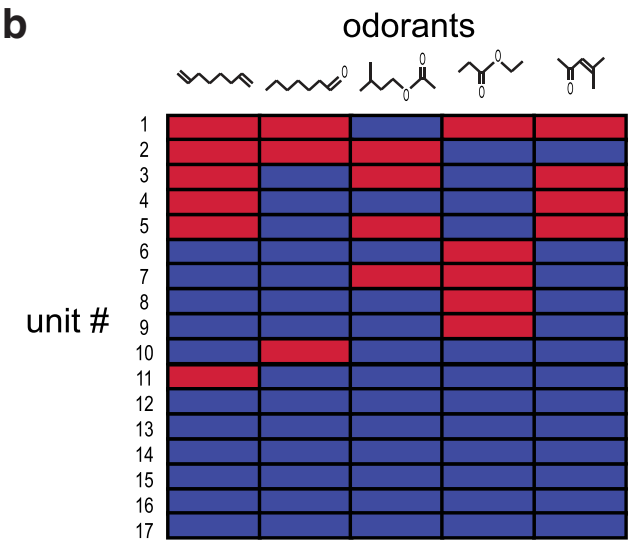

odor mixtures

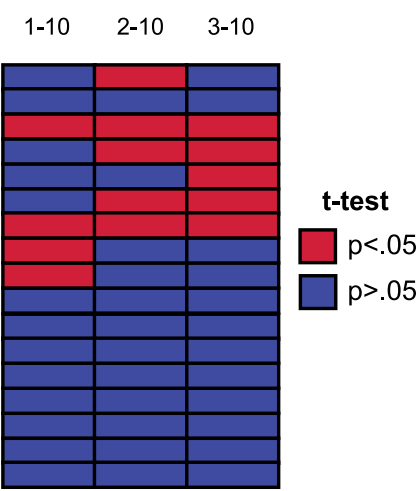

Figure 4. Odorant responsivity of tubercle units. $\boldsymbol{a}$, Spike raster plots and peristimulus time histograms from two units (2 separate mice) throughout multiple presentations with five different odorants (gray-shaded regions). The top halves of each panel shows spike activity as raster plots (dots) of single units in response to each of the five odorants across multiple trials ( $y$-axis; each row is a single trial). Summarizing each raster plot is a peristimulus time histogram with average firing rate (in hertz) on the $y$-axis. Odorants are as follows (from left to right): 1,7-octadiene, ethyl proprionate, heptanal, isoamyl acetate, and 4-methyl-3-penten-2-one. Histograms, $100 \mathrm{~ms}$ time bins. $\boldsymbol{b}$, Statistical summary of odor selectivity of 17 tubercle units in response to the five odorants outlined in $\boldsymbol{a}$ and three odorant mixtures (right matrix) (see Materials and Methods). The red cells indicate significant responses across all trials ( $p<0.05$, two-tailed $t$ test, $2 \mathrm{~s}$ prestim vs $2 \mathrm{~s}$ during stim). Units 6 and 2, Same data as in $\boldsymbol{a}$. Whereas some units (e.g., 1 and 3) responded to all but one or two odorants, other units (e.g., 11) responded to only one odorant.

and Katz, 2007) and layer II/III pyramidal cells of the PCX (Wilson, 2001; Rennaker et al., 2007; Yoshida and Mori, 2007; Poo and Isaacson, 2009) are capable of discriminating between odorants. However, whether tubercle units also discriminate odorants is unknown. Therefore, we recorded odor-evoked responses to a panel of five monomolecular odorants (see Materials and Methods) in 17 units ( $n=11$ mice). Odors were presented in a counterbalanced manner, for a minimum of four trials each.

We found that olfactory tubercle single units show robust responses to odorants. In particular, 64\% (11 of 17) of olfactory tubercle units showed significant responses to at least one of the five odorants ( $p<0.05$ for each odor/unit; $\geq 4$ trials/odor; two-tailed $t$ test). In the example shown in Figure $4 a$, the unit on top (unit 6) responds selectively to a single odorant (ethyl propionate). The unit beneath (unit 2), however, has a broader receptive range and significantly responds to three of the five odorants (Fig. 4a). We also presented the same 17 units with overlapping, complex odorant mixtures-all sharing similar components yet with some omitted (Barnes et al., 2008). These odorant mixtures were presented in a counterbalanced manner along with the odorants used in Figure $4 a$. Of 17 olfactory tubercle units, 8 (47\%) showed significant responses to at least one of the three odorant mixtures $(p<0.05$ for each mixture/unit; $\geq 4$ trials/mixture). Of the eight units that significantly responded to a mixture, only two (20\%) showed significant responses to all three mixtures (Fig. $4 b$ ). More commonly, a single unit responded to just a single mixture (Fig. $4 b$ ) — even though all three mixtures shared overlapping components. These data suggest that olfactory tubercle single units are capable of discretely responding to individual odorants.

\section{Auditory evoked responses in the tubercle}

We next examined whether the olfactory tubercle responds to auditory stimuli. Auditory-associated fiber projections into early olfactory processing areas originate within the hippocampus (Deadwyler et al., 1987), the ventral pallidum (Budinger et al., 2006), and even the primary auditory cortex itself (Budinger et al., 2008). To test the hypothesis that olfactory tubercle units are sensitive to auditory input, we presented anesthetized mice with a 2 s auditory stimulus (tone) via a simple piezo speaker during simultaneous extracellular recordings.

Tones evoked responses at both the population (LFP) and singleunit level in the olfactory tubercle (Fig. 5). In the example shown in Figure $5 a$, a tone (onset timed to the respiration cycle as in Figs. 3 and 4) evoked an increase in spike rate across multiple trials. We screened a total of 26 isolated single units for tone-evoked responses. Five of the 26 units $(>19 \%)$ showed significant responses to tone presentation (Fig. $5 c$ ) ( $p<0.05$ for each unit; $\geq 4$ tone trials/unit; two-tailed $t$ test). Furthermore, LFP power spectrum analysis revealed that, whereas tone presentation did not significantly increase theta $\left(t_{(9)}=\right.$ $-1.36 ; p=0.20)$ oscillations, beta $\left(t_{(24)}=-11.0 ; p<0.0001\right)$ and gamma $\left(t_{(28)}=-7.36 ; p<0.0001\right)$ oscillations were both enhanced with tone (Fig. $5 d$ ). Although we limited our analysis of auditory 
a

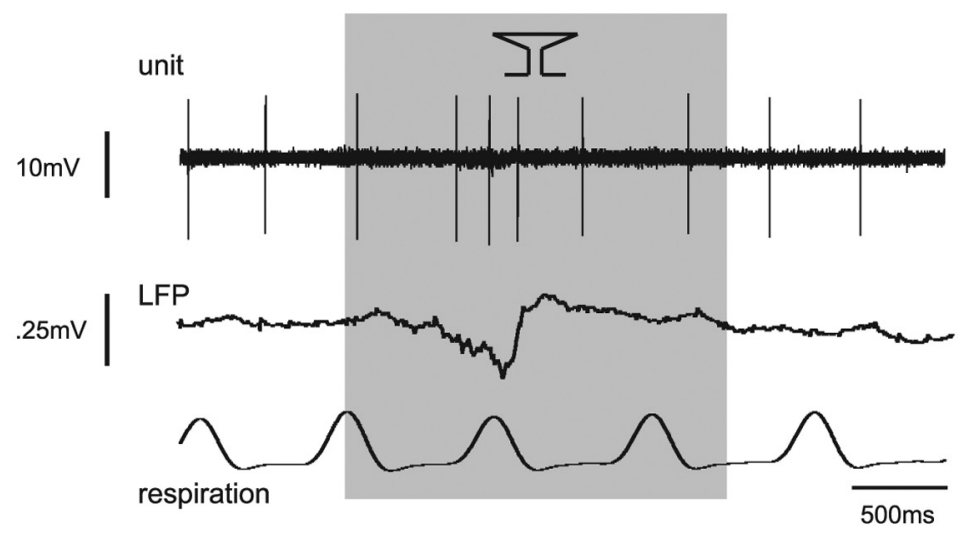

b
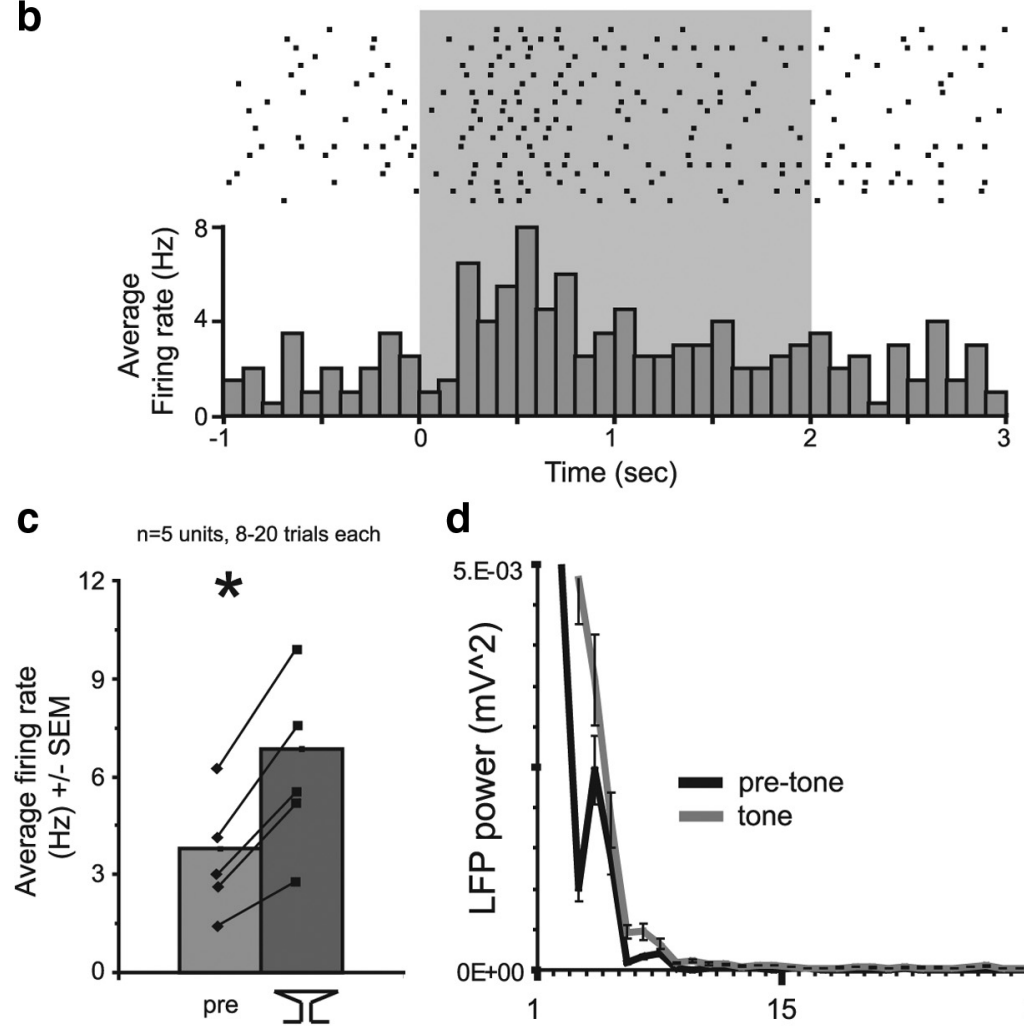

d

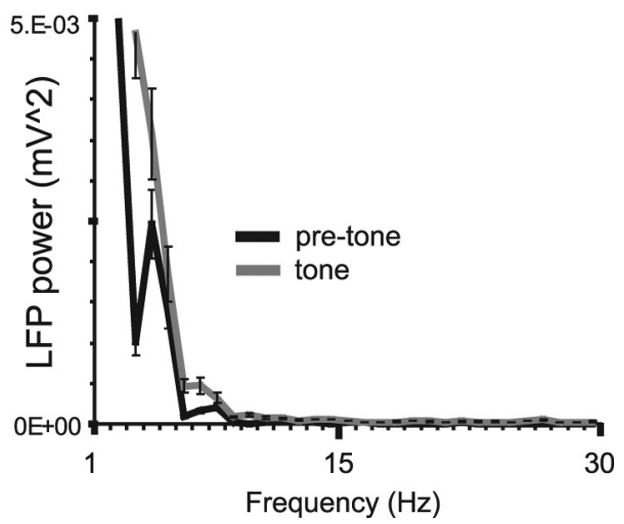

Figure 5. Tubercle units respond to an auditory stimulus. $\boldsymbol{a}$, Representative traces showing multiunit activity, LFP $(0-100 \mathrm{~Hz}$ second-order low-pass filter), and respiration over the course of $4 \mathrm{~s}$. Inhalation in respiratory trace is an upward deflection. Time of tone presentation is indicated by the shaded gray box. $\boldsymbol{b}$, Spike raster plot and peristimulus time histogram from a single unit throughout multiple presentations of an auditory tone. The top halves of each panel show spike activity as raster plots (dots) of the unit in response to the tone across multiple trials ( $y$-axis; each row is a single trial). Summarizing the raster plot is a peristimulus time histogram with average firing rate (in hertz) on the $y$-axis. Histogram, 100 ms time bins. c, Bar graph and scatter plot showing average "pre" (2 s prestim) and tone evoked (tone icon; 2 s during stim) responses of five tubercle units that all showed significant spike rate increases in response to tone. ${ }^{*} p<0.05$, two-tailed $t$ test. $\boldsymbol{d}$, LFP power spectrograms of pretone ( $2 \mathrm{~s}$ prestim) and tone ( 2 s during tone) LFP activity. Data are from four mice, five tone trials each.

stimuli to a simple tone for the purposes of this study, we also informally observed that alternative auditory stimuli (i.e., manual clapping, electronic buzzer) also evoked unit-level changes in activity (data not shown). These data demonstrate the functional input of auditory information into the olfactory tubercle.

\section{Olfactory-auditory modulation in the olfactory tubercle}

The above data show, for the first time, that single units in the olfactory tubercle respond to both olfactory and auditory input. Therefore, finally, to provide a test of olfactory-auditory modu- lation in the olfactory tubercle, we presented a subset of units $(n=17)$ with a temporally overlapping odor and tone (odor plus tone) to ask whether the presence of a stimulus in one modality affected the response to the other. The onset of both stimuli was timed off of the animal's respiratory signal as done previously for each stimulus (Figs. 3-5) (see Materials and Methods). LFP and unit traces for an odor, tone, and the overlapping odor plus tone are shown in Figure $6 a$. As reported in Figures 3 and 4, odor presentation evoked responses phasic with each respiration cycle. Also, similar as displayed in Figure 5, a tone elicited a brief burst of action potentials (Fig. $6 a$, middle). Finally, in this example, simultaneous presentation of odor plus tone resulted, on average, in a modest supraadditive effect (Fig. $6 a$, bottom trace; $b$ ). As displayed in Figure $6 b$, across all 17 units, the average evoked response for the odor was greater than that of the tone $\left(t_{(352)}=-2.64 ; p<0.01 ; n=353\right.$ total trials). Odor-plus-tone presentation on average elicited responses greater than that of the tone $\left(t_{(353)}=-2.97 ; p<0.005 ; n=355\right.$ total trials) but not of odor $\left(t_{(363)}=-1.49\right.$; $p>0.05 ; n=365$ total trials) alone. Similar to that of the odor presentation alone (Fig. $3 c$ ), odor plus tone elicited a significant change in beta $\left(t_{(24)}=-8.19 ; p<0.0001\right)$ and gamma $\left(t_{(28)}=-8.10 ; p<0.0001\right)$ oscillatory activity compared with the $2 \mathrm{~s}$ prestimulus (Fig. 6c). No changes in theta oscillations were observed in response to odor plus tone $\left(t_{(9)}=-1.98 ; p=0.07\right)$.

As shown in Figure 7, olfactory tubercle single units possessed diverse responses toward not only the odor or tone alone, but also to the odor-plus-tone stimulus. It is likely that averaging across all units, as done in Figure $6 b$, presents a less-than-representative image of the cross-modal functions within the olfactory tubercle. Looking at the response of individual units, a subpopulation $(>29 \%)$ of olfactory tubercle single units displayed cross-modal modulation in response to odor plus tone. For example, one unit showed a significant response to tone, but not odor, and showed response suppression to odor plus tone (Fig. 7, “\#”). In another example, a unit (Fig. 7, “ઋ”) failed to show significant responses to either tone or odor alone, but was significantly excited by odor plus tone. Thus, particular subpopulations of units in the olfactory tubercle functionally display olfactory-auditory convergence and cross-modal modulation.

\section{Absence of olfactory-auditory convergence in upstream OB neurons}

The data so far suggest that a population of olfactory tubercle units are capable of olfactory-auditory convergence. This effect 

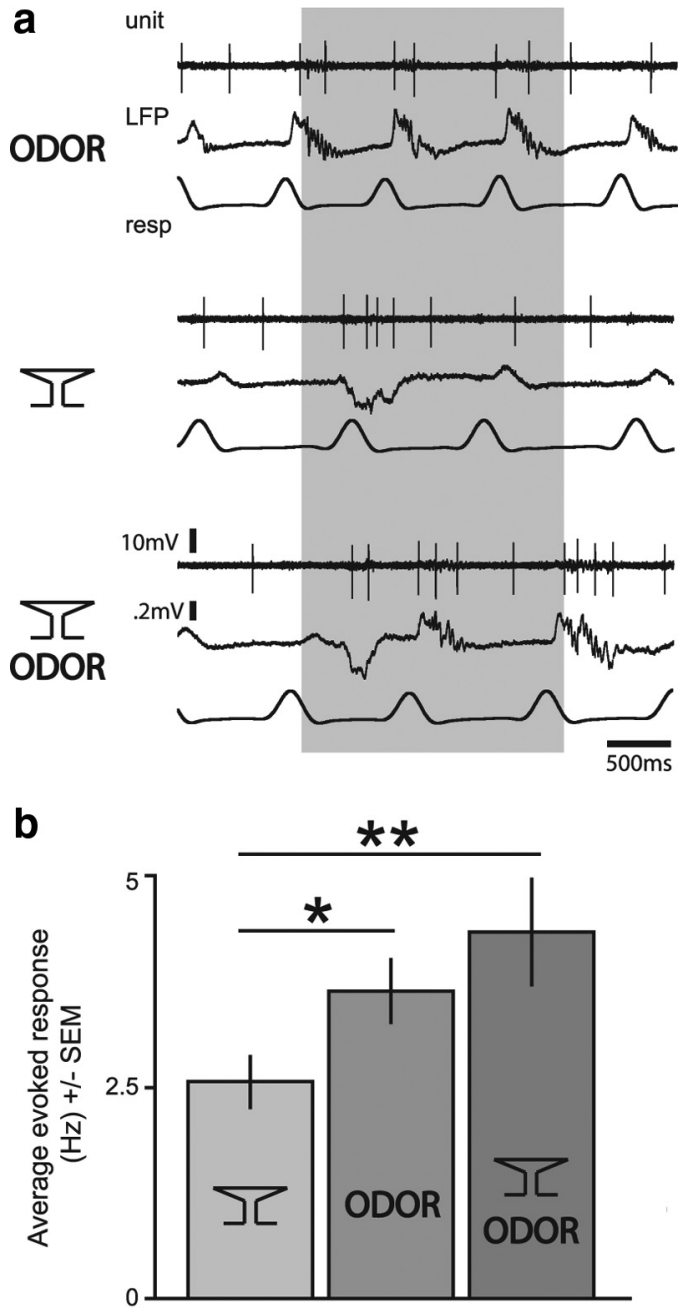

\section{C}

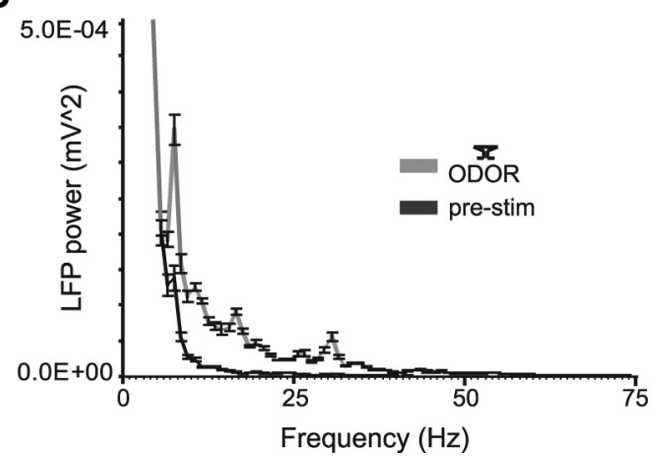

Figure 6. Olfactory-auditory interactions in olfactory tubercle single units. $\boldsymbol{a}$, Representative traces showing multiunit activity, LFP ( $0-100 \mathrm{~Hz}$ second-order low-pass filter), and respiration over the course of 4 s relative to stimulus onset. Inhalation in respiratory trace is an upward deflection. Time of stimulus presentation is indicated by the shaded gray box. Data shown are from a single electrode location (same mouse) and reflect events in response to an odor ("ODOR"; top panel), auditory tone (tone icon; middle panel), and simultaneous tone and odor presentation (tone icon and "odor"; bottom panel). Histograms, 100 ms time bins. b, Average evoked response (2 s stim to 2 s prestim), for each tone, odor, and odor-plus-tone stimulus ( $n=17$ units, 173-184 trials/ stimulus). ${ }^{*} p<0.01$, tone versus odor; ${ }^{* *} p<0.005$, tone versus odor plus tone, two-tailed $t$ test. $c$, LFP power spectrograms of prestim ( $2 \mathrm{~s}$ prestim) and odor-plus-tone ( $2 \mathrm{~s}$ during odor plus tone) LFP activity. Data are from four mice, four trials each. Error bars indicate SEM.

may be attributable to the direct convergence of both olfactory and auditory input at the level of the tubercle. Alternatively, convergence may arrive upstream (within the $\mathrm{OB}$ ), which is simply reflected within the olfactory tubercle. Therefore, finally, we ex- amined whether upstream MTs within the OB show responses to a tone.

We performed extracellular MT single-unit and/or multiunit recordings in an additional set of experiments $(n=5$ mice, 20 units). Similar to that done for olfactory tubercle recordings (Figs. 6, 7), MT unit responses were assessed in the presence of a tone, an odor, or an odor-plus-tone stimulus ( $n=5-15$ trials per stimulus per unit) (see Materials and Methods). As shown in Figure 8, $a$ and $b$, individual MTs showed responses to the odor stimulus $\left(t_{(214)}=-10.47 ; p<0.0001\right)$ and to the odor-plus-tone stimulus $\left(t_{(214)}=-9.89 ; p<0.0001\right)$, yet failed to respond to the tone alone $\left(t_{(214)}=-1.49 ; p>0.05\right)$. In fact, 0 of 20 of the MT units showed a significant response to tone $(p>0.05$ for each unit; $\geq 5$ tone trials/unit; two-tailed $t$ test). Furthermore, the evoked responses of odor-plus-tone stimuli did not significantly differ from that of odor alone (Fig. $8 b)\left(t_{(214)}=-0.02 ; p>0.05\right)$. This was true even at the single-unit (vs group) level. Indeed, unlike in the tubercle, no individual MT units showed significant differences in evoked responses between odor and odor plus tone ( $p>0.05$ for each unit; $\geq 5$ trials/unit; two-tailed $t$ test) (data not shown). Thus, whereas units within the $\mathrm{OB}$ and the olfactory tubercle both represent odors (Figs. 3, 8a), olfactory tubercle units respond to both olfactory and auditory information.

\section{Discussion}

To date, the majority of research on the olfactory tubercle has emphasized its apparent role in reward circuitry (for review, see Ikemoto, 2007). In contrast, despite its vast interconnectedness (Haberly and Price, 1977; Schwob and Price, 1984; Johnson et al., 2000), surprisingly little attention has been devoted to this relatively large region with regard to its functional role in sensory processing. Here, we used extracellular recordings in anesthetized mice to examine a previously unexplored role of the olfactory tubercle-cross-modal sensory convergence. We found that single units in the olfactory tubercle not only show selective odor responses but also demonstrate apparent convergence of olfactory and auditory inputs with single units displaying responses to both odors and tones. Remarkably, some units displayed crossmodal modulation, with supraadditive or suppressive responses to the simultaneous presentation of odor and tone. Thus, the tubercle serves cross-modal functions, likely binding smells and sounds early in the olfactory processing stream.

\section{Olfactory coding in the olfactory tubercle}

Projections from the OB target a wide range of secondary olfactory regions, including the olfactory tubercle. MTs of the OB fasciculate to form the lateral olfactory tract, which travels along the ventral-lateral aspect of the brain. Anterior to, and around the same anatomical locations at which MTs project into the anterior PCX, MTs project into olfactory tubercle layer I and synapse onto primary tubercle principal neurons. The olfactory tubercle has received little attention in attempts to understand its functional roles in olfactory coding (but see McNamara et al., 2004; Chiang and Strowbridge, 2007; Carriero et al., 2009). Indeed, to our knowledge, only one study (Murakami et al., 2005) has recorded odor-evoked unit responses in vivo. What we do know about the olfactory-processing function of the olfactory tubercle is that olfactory sensory input from the $\mathrm{OB}$ can arrive in the tubercle via the lateral olfactory tract or via the PCX (Carriero et al., 2009). Voltage-sensitive dye imaging from in vitro guinea pig brains showed that the olfactory tubercle is homogeneously activated by lateral olfactory tract stimulation in a biphasic manneryet with slightly differing temporal dynamics (Carriero et al., 


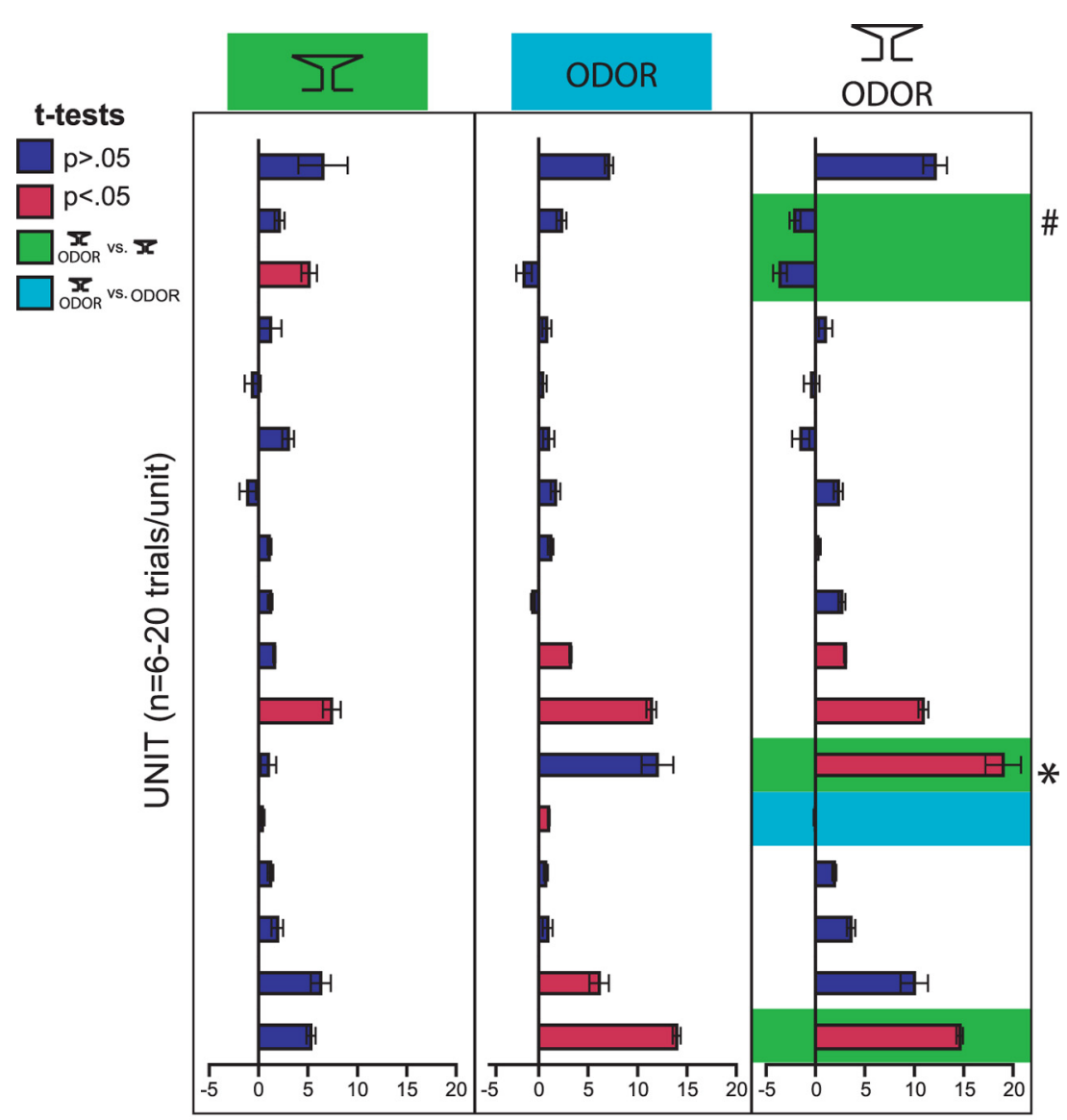

Average evoked response (stim - 2sec pre-stim) +/- SEM

Figure 7. Subpopulations of tubercle single units show olfactory-auditory cross-modal modulation. Bar graphs of average evoked responses across 17 units (different from those in Fig. 4), in response to tone (left), odor (middle) (for odor, see Materials and Methods), and simultaneous tone and odor presentations (right column). The red bars indicate significant responses of that unit across all trials ( $p<0.05$, two-tailed $t$ test, 2 s prestim vs $2 s$ during stim). The blue bars are insignificant $(p>0.05)$. The green background color behind bars indicates that response of that unit is significantly different from responses of that unit to tone alone ( $p<0.05$, two-tailed $t$ test). The blue background color behind bars indicate that response of that unit is significantly different from responses of that unit to odor alone ( $p<0.05$, two-tailed $t$ test). Whereas some units (e.g., indicated with "**") showed supraadditive responses to both stimuli simultaneously, others (e.g., indicated with "\#") showed suppressive responses. Error bars indicate SEM.

2009). Activity spreads throughout the tubercle from lateral to medial-likely reflecting the MT input along the lateral edge (Carriero et al., 2009). Additional experiments by the same group revealed that severing the lateral olfactory tract projection into the PCX reduced the secondary biphasic response of the tubercle. Indeed, the tubercle is interconnected with the PCX and tenia tecta by a large association fiber network (Luskin and Price, 1983). Thus, olfactory information can enter the tubercle by two routes: directly from the OB via MTs or by PCX association fibers.

Here, in agreement with the standing anatomical (Haberly and Price, 1977; Schwob and Price, 1984; Johnson et al., 2000) and functional evidence (Murakami et al., 2005; Chiang and Strowbridge, 2007; Carriero et al., 2009), we found that the olfactory tubercle responds to odors. On the population level, olfactory tubercle LFPs showed robust odor-evoked oscillations-reflecting global activation of the olfactory tubercle network by individual odors. Furthermore, on a single-unit level, odor-responsive units were readily observed - with almost $70 \%$ of single units responsive to at least one of the five odorants we tested. Notably, screening responses to an even larger battery of odorants (including aldehydes and ketones, for example) may have resulted in a greater portion of units responsive to odorants. Additionally, although not a focus of the present study, LFP and single-unit activity displayed entrainment to the respiratory rhythm. Given the importance of respiratory behavior on shaping the neural code for odors, future studies into respiratory coupling of odor information, especially in relation with PCX and/or OB activity, will provide useful information on the coherence between these primary structures in conveying the early olfactory code.

\section{Mechanisms of olfactory-auditory cross-modal convergence}

Previous studies have uncovered olfactory-visual (Gottfried and Dolan, 2003) and olfactory-gustatory cross-modal convergence and integration (for review, see Verhagen and Engelen, 2006). In the present studies, we explored the olfactory tubercle for the existence of olfactory-auditory convergence. We found that $\sim 20 \%$ of tubercle units were significant driven by an auditory tone. In contrast, $0 \%$ of upstream neurons recorded in the $\mathrm{OB}$ displayed significant tone-evoked responses. In the present study, a single tone and intensity was tested, thus whether and how the olfactory tubercle may code for auditory stimuli of differing intensities or frequencies will be an interesting avenue for future research. Furthermore, and especially relevant to the present study is whether olfactory-auditory interactions in the tubercle depend on particular auditory stimuli. For instance, it is possible that spike rate in the olfactory tubercle is proportionally related with tone intensity. Finally, whether the olfactory tubercle acts alone or facilitates olfactory-auditory cross-modal interactions with other regions remains to be explored. For example, it is unclear whether other olfactory cortical areas also show crossmodal convergence like that shown here in the tubercle. Furthermore, the auditory cortex and possibly other olfactory processing areas may serve a role in olfactory-auditory cross-modal convergence (Budinger and Scheich, 2009). Indeed, auditory cortex evoked potential (Halene et al., 2009) and single-unit responses are modulated by olfactory cues in behaving rats (Otazu et al., 2009). Despite these issues, the present results clearly demonstrate that the single units in the olfactory tubercle bind information from both olfactory and auditory sensory channels.

Previous work in the olfactory system has shown extramodal stimuli are capable of nonspecific modulating olfactory pathway activity (Gray et al., 1986; Wilson and Sullivan, 1990; Bouret and Sara, 2002). Both the unit selectivity (only $\sim 20 \%$ of units responded to tone) and temporal precision of auditory-evoked spikes in the tubercle argue that these responses are truly crossmodal convergence, and not the result of nonspecific neuromodulatory influences. Based on previous work (Wilson and Sullivan, 1990; Bouret and Sara, 2002), such responses, if caused by 
neuromodulatory influences, may likely be more prevalent in the tubercle and likely would last for longer durations.

\section{Cognitive and behavioral implications for olfactory-auditory convergence}

The olfactory tubercle is part of the mesocorticolimbic pathway. Previous work has suggested that increased dopaminergic activity throughout this pathway, including within the olfactory tubercle, facilitates changes in behavioral strategies (Koob et al., 1978; Oades, 1981). Therefore, it is possible that the olfactory-auditory convergence and modulation in the olfactory tubercle may play a role in rapid behavioral plasticity in arousing situations. For instance, if a mouse hears the sound of an approaching predator's paw steps, this may heighten responsiveness of olfactory tubercle units to predator odor. The location of the olfactory tubercle in the mesocorticolimbic pathway positions this newly integrated information in an ideal manner for the rapid, arousal-related behavioral responses required to flee the oncoming predator. Thus, in general, the olfactory tubercle may serve as a site for not only olfactory arousal-related decision making, but also odor orientation and localization. In support of this, recent work has demonstrated that rats increase their sniffing frequency-a behavior normally implicated in olfactory behaviors-during novel tone investigation (Harrison, 1979) and during a simple auditory discrimination task (Wesson et al., 2009).

Finally, intrinsic modulation of olfactory codes based on auditory input may underlie clinical reports of olfactory-auditory synesthesia (Simpson and McKellar, 1955) and possibly the ability for persons to relate auditory pitch with odor perceptual quality (Belkin et al., 1997). Although our results demonstrate for the first time that the olfactory tubercle is a site for olfactory and auditory convergence and that such activity is susceptible to cross-modal influences, whether and how these cross-modal influences may be altered based on cognitive task demands and levels of arousal is unknown.

\section{References}

Barnes DC, Hofacer RD, Zaman AR, Rennaker RL, Wilson DA (2008) Olfactory perceptual stability and discrimination. Nat Neurosci 11:1378-1380.

Belkin K, Martin R, Kemp SE, Gilbert AN (1997) Auditory pitch as a perceptual analogue to odor quality. Psychol Sci 8:340-342.

Bouret S, Sara SJ (2002) Locus coeruleus activation modulates firing rate and temporal organization of odour-induced single-cell responses in rat piriform cortex. Eur J Neurosci 16:2371-2382.

Budinger E, Scheich H (2009) Anatomical connections suitable for the direct processing of neuronal information of different modalities via the rodent primary auditory cortex. Hear Res 258:16-27.

Budinger E, Heil P, Hess A, Scheich H (2006) Multisensory processing via early cortical stages: connections of the primary auditory cortical field with other sensory systems. Neuroscience 143:1065-1083.

Budinger E, Laszcz A, Lison H, Scheich H, Ohl FW (2008) Non-sensory cortical and subcortical connections of the primary auditory cortex in Mongolian gerbils: bottom-up and top-down processing of neuronal information via field AI. Brain Res 1220:2-32. b

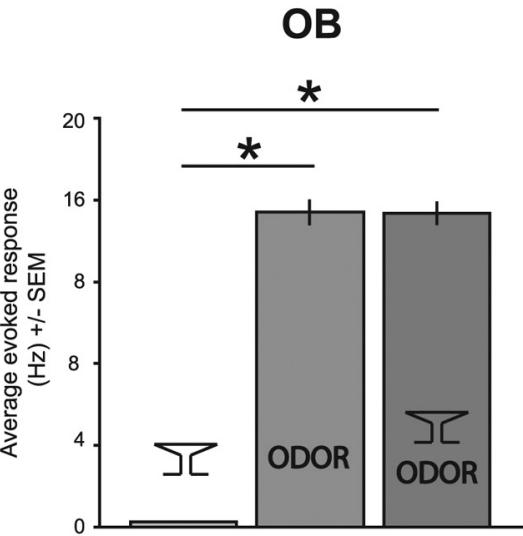

$\mathrm{OB}$

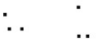
$\because \cdot$
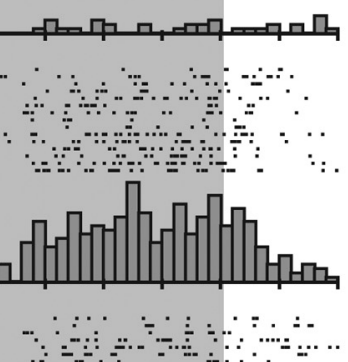

-
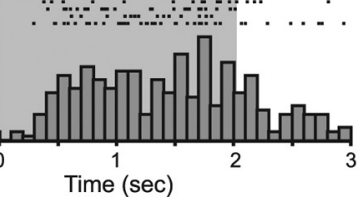

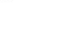

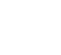

Figure 8. Olfactory-auditory convergence is not detected upstream of the olfactory tubercle. $\boldsymbol{a}$, Spike raster plots and peris(a) stim to 2 s prestim), for each tone, odor, and odor-plus-tone stimulus ( $n=20$ units, $\geq 108$ trials/stimulus). ${ }^{*} p<0.0001$, tone versus odor and tone versus odor plus tone, two-tailed $t$ test. Error bars indicate SEM.

Calvert GA, Spence C, Stein BE, eds (2004) The handbook of multisensory processes, Ed 1. Cambridge, MA: MIT.

Cang J, Isaacson JS (2003) In vivo whole-cell recording of odor-evoked synaptic transmission in the rat olfactory bulb. J Neurosci 23:4108-4116.

Carriero G, Uva L, Gnatkovsky V, de Curtis M (2009) Distribution of the olfactory fiber input into the olfactory tubercle of the in vitro isolated guinea pig brain. J Neurophysiol 101:1613-1619.

Cenier T, Amat C, Litaudon P, Garcia S, Lafaye de Micheaux P, Liquet B, Roux S, Buonviso N (2008) Odor vapor pressure and quality modulate local field potential oscillatory patterns in the olfactory bulb of the anesthetized rat. Eur J Neurosci 27:1432-1440.

Chiang E, Strowbridge BW (2007) Diversity of neural signals mediated by multiple, burst-firing mechanisms in rat olfactory tubercle neurons. J Neurophysiol 98:2716-2728.

Davis M (1989) Sensitization of the acoustic startle reflex by footshock. Behav Neurosci 103:495-503.

Davison IG, Katz LC (2007) Sparse and selective odor coding by mitral/ tufted neurons in the main olfactory bulb. J Neurosci 27:2091-2101.

Deadwyler SA, Foster TC, Hampson RE (1987) Processing of sensory information in the hippocampus. CRC Crit Rev Clin Neurobiol 2:335-355.

Freeman WJ (1978) Spatial properties of an EEG event in the olfactory bulb and cortex. Electroencephalogr Clin Neurophysiol 44:586-605.

Gottfried JA, Dolan RJ (2003) The nose smells what the eye sees: crossmodal visual facilitation of human olfactory perception. Neuron 39:375-386.

Gray CM, Freeman WJ, Skinner JE (1986) Chemical dependencies of learning in the rabbit olfactory bulb: acquisition of the transient spatial pattern change depends on norepinephrine. Behav Neurosci 100:585-596.

Haberly LB, Price JL (1977) The axonal projection patterns of the mitral and tufted cells of the olfactory bulb in the rat. Brain Res 129:152-157.

Haberly LB, Price JL (1978) Association and commissural fiber systems of the olfactory cortex of the rat. I. Systems originating in the piriform cortex and adjacent areas. J Comp Neurol 178:711-740.

Halene TB, Talmud J, Jonak GJ, Schneider F, Siegel SJ (2009) Predator odor modulates auditory event-related potentials in mice. Neuroreport 20:1260-1264.

Harrison JM (1979) The control of responding by sounds: unusual effect of reinforcement. J Exp Anal Behav 32:167-181.

Ikemoto S (2007) Dopamine reward circuitry: two projection systems from 
the ventral midbrain to the nucleus accumbens-olfactory tubercle complex. Brain Res Rev 56:27-78.

Johnson DM, Illig KR, Behan M, Haberly LB (2000) New features of connectivity in piriform cortex visualized by intracellular injection of pyramidal cells suggest that "primary" olfactory cortex functions like "association" cortex in other sensory systems. J Neurosci 20:6974-6982.

Kay LM, Laurent G (1999) Odor- and context-dependent modulation of mitral cell activity in behaving rats. Nat Neurosci 2:1003-1009.

Kay LM, Beshel J, Brea J, Martin C, Rojas-Líbano D, Kopell N (2009) Olfactory oscillations: the what, how and what for. Trends Neurosci 32:207-214.

Koob GF, Riley SJ, Smith SC, Robbins TW (1978) Effects of 6-hydroxydopamine lesions of the nucleus accumbens septi and olfactory tubercle on feeding, locomotor activity, and amphetamine anorexia in the rat. J Comp Physiol Psychol 92:917-927.

Luskin MB, Price JL (1983) The topographic organization of associational fibers of the olfactory system in the rat, including centrifugal fibers to the olfactory bulb. J Comp Neurol 216:264-291.

McNamara AM, Cleland TA, Linster C (2004) Characterization of the synaptic properties of olfactory bulb projections. Chem Senses 29:225-233.

Murakami M, Kashiwadani H, Kirino Y, Mori K (2005) State-dependent sensory gating in olfactory cortex. Neuron 46:285-296.

Nagayama S, Takahashi YK, Yoshihara Y, Mori K (2004) Mitral and tufted cells differ in the decoding manner of odor maps in the rat olfactory bulb. J Neurophysiol 91:2532-2540.

Oades RD (1981) Dopaminergic agonistic and antagonistic drugs in the ventral tegmentum of rats inhibit and facilitate changes of food-search behaviour. Neurosci Lett 27:75-80.

Otazu GH, Tai L-H, Yang Y, Zador AM (2009) Engaging in an auditory task suppresses responses in auditory cortex. Nat Neurosci 12:646-654.

Paxinos G, Franklin K (2000) The mouse brain in stereotaxic coordinates, Ed 2. San Diego: Academic.

Piesse G (1857) The art of perfumery, and methods of obtaining the odors of plants. Philadelphia: Lindsay and Blakiston.

Poo C, Isaacson JS (2009) Odor representations in olfactory cortex: "sparse" coding, global inhibition, and oscillations. Neuron 62:850-861.

Rankin CH, Abrams T, Barry RJ, Bhatnagar S, Clayton DF, Colombo J, Cop- pola G, Geyer MA, Glanzman DL, Marsland S, McSweeney FK, Wilson DA, Wu C-F, Thompson RF (2009) Habituation revisited: an updated and revised description of the behavioral characteristics of habituation. Neurobiol Learn Mem 92:135-138.

Rennaker RL, Chen CF, Ruyle AM, Sloan AM, Wilson DA (2007) Spatial and temporal distribution of odorant-evoked activity in the piriform cortex. J Neurosci 27:1534-1542.

Rinberg D, Koulakov A, Gelperin A (2006) Sparse odor coding in awake behaving mice. J Neurosci 26:8857-8865.

Schwob JE, Price JL (1984) The development of axonal connections in the central olfactory system of rats. J Comp Neurol 223:177-202.

Scott JW, McBride RL, Schneider SP (1980) The organization of projections from the olfactory bulb to the piriform cortex and olfactory tubercle in the rat. J Comp Neurol 194:519-534.

Simpson L, McKellar P (1955) Types of synaesthesia. J Ment Sci 101: 141-147.

Smith JJ, Shionoya K, Sullivan RM, Wilson DA (2009) Auditory stimulation dishabituates olfactory responses via noradrenergic cortical modulation. Neural Plast 2009:1-6.

Verhagen JV, Engelen L (2006) The neurocognitive bases of human multimodal food perception: sensory integration. Neurosci Biobehav Rev 30:613-650.

Wesson DW, Verhagen JV, Wachowiak M (2009) Why sniff fast? The relationship between sniff frequency, odor discrimination, and receptor neuron activation in the rat. J Neurophysiol 101:1089-1102.

Wilson DA (1998) Habituation of odor responses in the rat anterior piriform cortex. J Neurophysiol 79:1425-1440.

Wilson DA (2001) Receptive fields in the rat piriform cortex. Chem Senses 26:577-584.

Wilson DA, Leon M (1988) Spatial patterns of olfactory bulb single-unit responses to learned olfactory cues in young rats. J Neurophysiol 59:1770-1782.

Wilson DA, Sullivan RM (1990) Olfactory associative conditioning in infant rats with brain stimulation as reward. I. Neurobehavioral consequences. Brain Res Dev Brain Res 53:215-221.

Yoshida I, Mori K (2007) Odorant category profile selectivity of olfactory cortex neurons. J Neurosci 27:9105-9114. 